\title{
Extraction of Total Polyphenols Kelp Optimization Test
}

\author{
Zhong Wang ${ }^{1}$, Yong-guang $\mathrm{Bi}^{2, *}$, Xue-wei Chen ${ }^{2}$, Xue-mei Liü ${ }^{2}$, Hai-lan Huang ${ }^{2}$ \\ ${ }^{1}$ School of Clinical Medicine, Guangdong Pharmaceutical University, Guangzhou 510006, Guangdong, China \\ ${ }^{2}$ School of Pharmacy, Guangdong Pharmaceutical University, Guangzhou 510006, Guangdong, China
}

\begin{abstract}
In this study, seaweed kelp as the raw material, the use of ultrasound-assisted method of extraction process kelp total polyphenols were discussed. Research on the single factor affecting the total polyphenol extraction: ultrasonic time, ultrasonic power, ultrasonic temperature, ethanol concentration. And the use of orthogonal experiment to optimize their processes, resulting from the visual analysis of optimum extraction process: The optimum conditions were: extraction time $4 \mathrm{~h}$, a temperature of 65 to ${ }^{\circ} \mathrm{C}$, ethanol concentration of $80 \%$. The results show that: the ultrasonic assisted extraction of total polyphenols kelp practical and simple and efficient method for the application of ultrasonic assisted extraction process chemical composition of algae provide references.
\end{abstract}

Plant polyphenols (plantpolyphenol), also known as tannins (tannins) are a class of widely present in plants polyphenols, mainly in the skin of plants, roots, wood, leaves, fruit sinks ${ }^{[1,2]}$. Plant polyphenols in nature reserves is very rich, as a class of natural compounds with biological activity, human nutrition and health has a significant impact. With further research, plant polyphenols in medicine, food, leather industry, household chemicals, etc ${ }^{[3-5]}$. have shown an increasingly important role. Plant polyphenols began in the 18th century. In the form of a mixture of its complex structure, chemically active, and often similar in nature to a lot of homologs exist, so before the 1930s, progress has been very slow chemical polyphenols. Scientific classification of plant polyphenols until 1920 was proposed by K.Freudenberg, that is, depending on the chemical structure, hydrolysis plant polyphenols into tannins and condensed tannins. The former is mainly orange acid and its derivatives and polyols vinegar bond or II bond formation, may be subdivided into tannins and trample flowers tannins categories; the latter is mainly light base flavanol monomer condensates CC bond between monomers linked. Tannin molecular weight is generally between 500-3000, which is the molecular weight range of condensed tannins, flavonoids now a molecular weight of less than 500 are also included in a class of plant polyphenols ${ }^{[6]}$. In the pharmaceutical, food, cosmetic applications, general plant polyphenols molecular weight 500-1500, and in industry, such as leather industry, uses a macromolecular polyphenols.

Kelp (Laminaria japonica), is a low-temperature waters in large marine brown algae growing plant, is a seaweed plant. Phaeophyceae seaweed, kelp Section. Sporophyte large, brown, flat ribbon, up to 20 meters. Divided blade, shank and holdfast, fixer was rhizoid shape. Leaf epidermis, cortex and pith tissue formed, the lower leaves have sporangium. Having mucus cavity secrete slippery substances. Holdfast tree branch, attached to the seabed rocks. Growth at a lower temperature of the sea $^{[7]}$. A large number of China's coastal and northern Zhejiang, Fujian coast cultivation, production in the world. Alginate and iodine-rich, edible and extract iodine, alginate, mannitol and other industrial raw materials.

Kelp in a long history in China, "Shen Nong's herbal classic", "Bencao Gangmu" and "food ".The matter had been recorded in . As early as 1400 years ago, the Chinese people gradually on the edible seaweed the understanding, such as "materia Sutra" cloud: "kelp, salty soft Kennedy"; "Materia Medica": "the kelp,male on algae, dysphagia with constant. The old cover removing phlegm; Meng Xian Tang Dynasty "with" therapeutic Materia Medica ":" kelp under the gas, long service "and so on, that thin kelp has expectorant, detumescence, slimming effect. Modern pharmacologyresearch has revealed that the study, medical and food nutrition has been revealed that the kelp has many biological activities, such as: drop

Blood glucose, blood lipids, blood pressure, anti coagulation, anti tumor, antibacterial, antiviral, immune regulation, anti radiation and so on, these functions are to a large extent with the physiological activity of the substance - polysaccharide related

The research on the pharmacological effects of Polysaccharide from kelp showed that the polysaccharide had many physiological activities, such as lowering blood sugar, lowering blood fat, lowering blood pressure, anti coagulation, anti tumor, anti-virus and so on. It could regulate the body's immunity and resist many kinds of diseases. Polysaccharide is a kind of natural macromolecular organic matter, which is a kind of natural macromolecular organic substance, which is a kind of biological activity. It is an indispensable and important component in organism. Due to different living environment in different sea areas, the active components of the polysaccharide in the body are also different, and the extraction process conditions are different from those in other areas. Existing research mainly concentrated in the sale of kelp, Fujian sea area, kelp, Zhejiang sea area, Dalian sea area, etc., and the South China Sea Kelp research has not been reported. But due to the low added value of traditional industries, the product is not processed or processed by the simple kelp products. Therefore, this paper takes the South China Sea Kelp polyphenols extraction as the research object, in order to open up a new way for the deep processing of kelp products and the efficient utilization of the kelp resources.

In this paper, ultrasound-assisted extraction process on kelp total polyphenols were studied to explore the four single factors on kelp total polyphenol extraction and orthogonal experiment to find the kelp with ultrasound assisted extraction of total polyphenols optimum 
extraction process, provide a reference for large-scale industrial applications.

\section{Materials and Methods}

\subsection{Test Materials}

Kelp: origin of Hainan Province, China; ethanol, petroleum ether, chloroform, ethyl acetate, n-butanol, gallic acid, sodium nitrite, aluminum nitrate, sodium hydroxide, sodium carbonate and other analytical reagents.

\subsection{Experimental Instrument}

Swing-speed grinder: Wenling Lin Machinery Co.; AY120 electronic analytical balance: Shimadzu Corporation; KH-400KDB High Power CNC ultrasonic cleaner: Ultrasonic Instrument Co., Ltd. Kunshan Wo Chong; SHZ-D (III) circulating water pumps: Gongyi City to Hua instrument Co., Ltd.; RE-52AA rotary

Tab.1 Orthogonal experiment factors and levels

\begin{tabular}{|c|c|c|c|c|c|c|c|}
\hline level & A & time/h & $\mathrm{B}$ & temperature $/{ }^{\circ} \mathrm{C}$ & C ethanol concentration /\% & $\mathrm{D}$ & Power/W \\
\hline 1 & & 2 & & 45 & 40 & & 300 \\
\hline 2 & & 3 & & 55 & 60 & & 300 \\
\hline 3 & & 4 & & 65 & 80 & & 300 \\
\hline
\end{tabular}

\subsubsection{Determination of total polyphenol}

Colorimetric determination of polyphenol content of potassium permanganate method, ferrous tartrate method, Prussian blue method, but potassium permanganate method has error, complicated operation and other shortcomings, ferrous tartrate method for determination of catechins substances with special properties, Prussian blue method is unstable data, errors and other shortcomings in the determination of polyphenols, while Folin reagent colorimetric method is commonly used measurement method plant polyphenolic compounds, with low cost, operation simple, accuracy and precision advantages, so this paper uses Folin reagent colorimetric determination of total polyphenols in kelp.

Referring Folin reagent colorimetric method to establish a standard curve: Precision Weigh gallic acid $5 \mathrm{mg}$, set $25 \mathrm{~mL}$ volumetric flask, add distilled water and diluted to the mark, shake, that give a concentration of gallic $0.2 \mathrm{mg} / \mathrm{mL}$ sub-acid solution, gallic acid lessons $0,0.1,0.2,0.3,0.4,0.5 \mathrm{~mL}$ of solution were placed in $10 \mathrm{~mL}$ volumetric flask, then add distilled water to each $6 \mathrm{~mL}$, Folin reagent $0.5 \mathrm{~mL}$, shake and let stand $5 \mathrm{~min}$, then each plus $20 \%$ sodium carbonate solution $1.5 \mathrm{~mL}$, diluted with distilled water to the mark, shaken, at room temperature for $30 \mathrm{~min}$, the absorbance was measured at $763 \mathrm{~nm}$ place. Gallic acid standard concentration c of the horizontal in order to determine the gallic acid solution absorbance A for the vertical axis, to prepare a standard curve (Figure 3). By regression statistics, it was the standard curve equation: $\mathrm{A}=95.60 \mathrm{c}+0.0063, \mathrm{R}^{2}=0.9998$. It indicates the amount of gallic acid in the $0 \sim 0$. 01mg/mLrange absorbance evaporator: Haiya Rong Biochemical instrument Factory.

\subsection{Experimental Methods}

\subsubsection{Sample Preparation}

The kelp after the use of clean water and dried, and pulverized with a pulverizer spare.

\subsubsection{Preliminary exploration of the sample extraction process}

Factors affecting extract yields have extraction time, extraction temperature, extract ethanol concentration. In order to determine these three integrated factors on kelp yield of flavonoids and polyphenols design L9 (33) orthogonal test ultrasonic extraction and determination. $10 \mathrm{~g}$ each experiment were taken to a solid-liquid kelp experimental operation (see Table 1) 1:10.

value good linear relationship. To learn of the prepared test sample solution $1.0 \mathrm{~mL}$, total polyphenol content was measured according to the method described above.

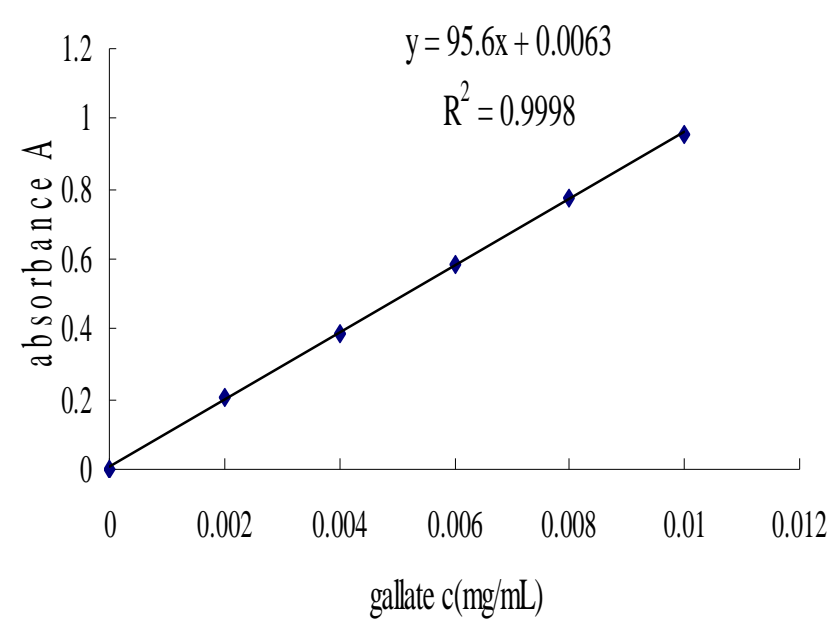

Fig.1 Gallic acid reference standard curve

\section{Results and analysis}

\subsection{Orthogonal test results and analysis}

orthogonal to optimize the extraction conditions, based on the study, experimental factor table and experimental results of univariate analysis (Tab.2). 
Tab.2 Ultrasonic extraction of total polyphenols factor level post test analysis table

\begin{tabular}{|c|c|c|c|c|c|}
\hline \multirow{2}{*}{ No. } & \multicolumn{4}{|c|}{ Factor } & \multirow{2}{*}{$\begin{array}{l}\text { Polyphenol } \\
\text { content /mg }\end{array}$} \\
\hline & A & B & $\mathrm{C}$ & $\mathrm{D}$ & \\
\hline 1 & 1 & 1 & 1 & 1 & 5.2 \\
\hline 2 & 1 & 2 & 2 & 2 & 5.8 \\
\hline 3 & 1 & 3 & 3 & 3 & 10.2 \\
\hline 4 & 2 & 1 & 2 & 3 & 5.8 \\
\hline 5 & 2 & 2 & 3 & 1 & 9.0 \\
\hline 6 & 2 & 3 & 1 & 2 & 6.6 \\
\hline 7 & 3 & 1 & 3 & 2 & 11.1 \\
\hline 8 & 3 & 2 & 1 & 3 & 6.0 \\
\hline 9 & 3 & 3 & 2 & 1 & 7.7 \\
\hline k1 & 21.2 & 22.1 & 18.4 & & \\
\hline k2 & 21.4 & 20.8 & 19.3 & & \\
\hline k3 & 24.8 & 24.5 & 30.3 & & \\
\hline $\mathrm{R}$ & 1.2 & 1.23 & 3.97 & & \\
\hline
\end{tabular}

From the analysis results in Table 2, the ethanol concentration of the poor of this factor, followed by the temperature, to the time. These three factors that affect the largest concentration of ethanol in the extraction of polyphenols time with minimal impact. In this experiment, parameter optimization based on optimization of process parameters for each factor C3B3A3, namely optimum conditions:Extraction time $4 \mathrm{~h}$, a temperature of 65 to ${ }^{\circ} \mathrm{C}$, ethanol concentration of $80 \%$.

Tab.3 Anova table

\begin{tabular}{cccccc}
\hline Variance Source & Squares & Freedom & Mean square error & F value & P Fvalue \\
\hline & & & & & \\
A & 0.025 & 2 & 0.013 & 4.596 & 0.179 \\
B & 0.022 & 2 & 0.011 & 3.990 & 0.200 \\
C & 0.287 & 2 & 0.144 & & 0.019 \\
Error blank column & 0.005 & 2 & 0.003 & 52593 & \\
& & & & & \\
\hline
\end{tabular}

concentration of $80 \%$.

Results from Table 3 that the variance, at the time, temperature, ethanol concentration of these three factors, the ethanol concentration was significant. Comprehensive Tables 2 and 3 are two optimization process, and consider saving materials and energy, in order to obtain a higher extraction capacity, optimum conditions were: extraction time $4 \mathrm{~h}$, a temperature of 65 to ${ }^{\circ} \mathrm{C}$, ethanol concentration was $80 \%$.

\section{3 conclusions}

The experiments indicate, using ultrasonic assisted extraction of total polyphenols in kelp is not only feasible, but also a simple process. The solvent used in this test is ethanol, the ethanol is relatively inexpensive, less toxic organic solvents, reduce due to the residual solvent affect the quality of the oil. Factors affecting ultrasonic extraction cubeba oil with ethanol concentration, ultrasonic time and ultrasonic temperature. Through intuitive analysis of orthogonal experiment showed that the test to determine the optimum conditions were:

extraction time $4 \mathrm{~h}$, a temperature of $65 \mathrm{t}^{\circ} \mathrm{C}$, ethanol

\section{Acknowledgements}

This work is supported by Guangdong Provincial Oceanic and Fishery Bureau - marine fisheries science and technology and industrial development projects (No. A201401C07).

\section{Reference}

[1] Nagasawa N, Mitomo H ,Yoslui F , Kume T.Radiation-induced degradation of sodium alginate [J]. Polymer Degradation and Stability. 2000.69(3): 279-285.

[2] Pisani P, Bray F, Parkin DM. Estimates of the world-wide prevalence of cancer for 25 sites in the adult population[J]. International Journal of Cancer, 2002, 97:72-81.

[3] Yan X. J.,Li X. C. ,Zhou C. X.et al. . Prevention of fish oil rancidity by phlorotannins from Sargassum kiellmanianum [J].Appl.Phvcol., 1996, 8:201-203 .

[4] Yan X. J., Li X. C. et al..Studies on extraction 
procedure and antioxidative activity of phlorotannins from Sargassum kjellmanianum [J].Chin. J .Oceanol. Limnol. 1997,15(1) :42-45.

[5] Yan X. J., Fang G. M. et al. .Studies on free radical scavenging activity in Chinese seaweeds [J].Chin.J.Oceanol. Limno1..1999, 17(3): 240-246.

[6] Koki Nagayama, Yoshitoshi Iwamura, Toshiyuki Shibata. Bactericidal activity of phlorotannins from the brown alga Ecklonia kurome[J]. Journal of Antimicrobial 